\title{
Radiophotoconductivité de couches épaisses composites à base d'oxyde de plomb : influence des traitements thermiques, mécaniques et du dopage
}

\author{
J. Moreau ( $\left.{ }^{1}\right)$ et M. Pham Thi $(2, *)$ \\ (1) Laboratoire de Chimie Physique du Solide, Ecole Centrale, 453 Grande Voie des Vignes, 92290 Chatenay- \\ Malabry, France \\ (2) Laboratoire Central de Recherches, Thomson-CSF, Domaine de Corbeville, 91401 Orsay Cedex, France
}

(Reçu le 22 décembre 1989, accepté le 4 mai 1990)

\begin{abstract}
Résumé. - La photoconductivité des couches composites sérigraphiées à base de monoxyde de plomb $(5 \times 5 \times 2 \mu \mathrm{m})$ et de résine époxy est étudiée. Le seuil de percolation est déterminé à $82 \%$ en poids de $\mathrm{PbO}$. Les principales caractéristiques de la décharge xérographique sont examinées. L'acceptance de charge d'une couche chargée par des charges négatives ou positives est respectivement de $-3000 \mathrm{~V}$ et $2000 \mathrm{~V}$. Le potentiel de contraste est égal à $324 \mathrm{~V}$ lorsqu'une couche de $200 \mathrm{~m}$ est irradiée par une dose de $140 \mathrm{mR}$ $\left(3 \times 10^{9}\right.$ photons $\left.\mathrm{X}\right)$. Deux types d'interface matériau/électrode sont obtenus : un comportement ohmique pour un champ électrique inférieur à $10 \mathrm{kV} / \mathrm{cm}$ et un comportement injectant au-dessus. Un traitement thermique peut augmenter la photosensibilité des couches tandis que les actions mécaniques la dégradent. Le dopage ne modifie pas de façon sensible la photoconductivité.
\end{abstract}

\begin{abstract}
The photoconductivity of silk-printed thick composite layers consisting of lead monoxide powder $(5 \times 5 \times 2 \mu \mathrm{m})$ and epoxy binder resin has been investigated. The percolation threshold has been determined as $82 \%$ by weight of $\mathrm{PbO}$. The important xerographic properties of charge acceptance, contrast potential and dark decay were studied. The charge acceptance of the layer were $-3000 \mathrm{~V}$ and $2000 \mathrm{~V}$ for negative and positive charges respectively. The contrast potential was $324 \mathrm{~V}$ when a $200 \mathrm{~m}$ thick composite layer was illuminated with a dose of $140 \mathrm{mR}\left(3 \times 10^{9}\right.$ photons $\left.\mathrm{X}\right)$. Two behaviours of the composite layer/electrode interface were observed : an ohmic effect in a low electric field region $(<10 \mathrm{kV} / \mathrm{cm})$ and an injection effect at higher electric fields. Thermal treatment enhances the photosensibility whereas mechanical treatment lowers it. The photoconductivity was not significantly modified by doping.
\end{abstract}

\section{Introduction.}

La possibilité de remplacer les films radiologiques par des systèmes de détection utilisant des écrans de grandes surfaces suscite un regain d'intérêt pour les matériaux photoconducteurs sensibles aux photons $\mathrm{X}$. Un des matériaux présentant le meilleur compromis sur le plan de la mise en ouvre et des propriétés potentielles est le monoxyde de plomb.

Les propriétés photoconductrices de $\mathrm{PbO}$ avaient été explorées en vue d'applications diverses comme les tubes télévision [1], ou l'électrophotographie [2]. En radiologie, le rendement photonique et la résolution spatiale des images impliquent l'utilisation de couches épaisses de quelques centaines de microns.

$\left(^{*}\right)$ à qui la correspondance doit être adressée.
Les techniques de dépôt en " couches épaisses " sont donc préférables aux techniques du vide.

Le monoxyde de plomb existe sous deux formes cristallographiques: la phase tétragonale $(\alpha)$ est stable à la température ambiante tandis que la phase orthorhombique $(\beta)$ apparaît au-dessus de $489{ }^{\circ} \mathrm{C}$, le composé conservant cette phase à la température ambiante. Comme la transition $\alpha-\beta$ est irréversible et affecte fortement la photoconduction [3], l'obtention par frittage de couches épaisses de $\mathrm{PbO}$ pur, bien densifiées paraît difficile. Par contre des couches photoconductrices peuvent être mises en œuvre par association de ce matériau photoconducteur avec une résine organique [4]. Il est cependant connu qu'un dopage convenable permet de déplacer la transition $\alpha / \beta$ vers les hautes températures [5], ouvrant ainsi la possibilité de réaliser des couches $\mathrm{PbO}$ photoconductrices frittées. 
Nous présentons ici l'étude de la photosensibilité de couches composites $\mathrm{PbO} /$ résine époxy et du gain en sensibilité des poudres en fonction des traitements thermiques et mécaniques subis. Le rôle des dopages est souligné.

\section{Expérimental.}

2.1 Elaboration DES COUCHES. - La poudre de $\mathrm{PbO}$, constituée de grains de $5 \mu \mathrm{m}$ (Johnson Matthey), est tamisée puis dispersée dans une résine époxy (Elycolite 3012). Le mélange $\mathrm{PbO} /$ résine est déposé par sérigraphie sur des substrats en aluminium ou en pyrex préalablement recouverts d'une électrode transparente d'oxyde d'étain dopé à l'indium (ITO). La couche formée est d'abord séchée à l'air ambiant puis à $60^{\circ}$ pendant plusieurs heures. Des épaisseurs variables sont obtenues par dépôts successifs. La concentration de $\mathrm{PbO}$ est choisie de façon à ce que la rhéologie du mélange soit adéquate pour l'opération de sérigraphie tout en maximisant le contact entre les grains dans la couche.

2.2 Méthodes de Mesures. - Les techniques de décharge xérographique et de mesure de photocourant en continu ont été utilisées pour la caractérisation des propriétés électriques des couches composites.

2.2.1 Source d'irradiation. - Le rayonnement $\mathrm{X}$ est émis par un générateur Battrix (Thomson CGR). La tension d'accélération est de $80 \mathrm{kV}$. Le spectre d'émission est filtré par une plaque d'aluminium de $3 \mathrm{~mm}$ d'épaisseur. Le spectre d'émission mesuré par un spectromètre Enertec ND 65 présente une bande centrée à $44,5 \mathrm{kV}$. Le débit de dose est de $7 \mathrm{mR} / \mathrm{s}$ $\left(1,5 \times 10^{8}\right.$ photon $\left.\mathrm{X} / \mathrm{s}\right)$. Pour varier les doses d'exposition, on augmente le temps d'exposition.

Le rayonnement visible est une lampe halogène $100 \mathrm{~W}$.

2.2.2 Décharge xéroradiographique. - La couche composite est chargée pendant quelques secondes par effet Corona à $6 \mathrm{kV}$ puis placée devant la sonde de l'électromètre pour la mesure du potentiel de surface. A cette position, l'échantillon reçoit alors le faisceau $X$ à travers la face arrière et le potentiel de surface chute brusquement. Notons que pour effacer les charges résiduelles, il est nécessaire d'éclairer l'échantillon avant chaque mesure par une lumière blanche puis de le laisser dans le noir pendant $1 \mathrm{~min}$.

Le potentiel de surface initial $V_{0}$ (juste après la charge) est appelé l'acceptance de charge. La décroissance dans le noir de ce potentiel en fonction du temps représente la courbe d'autodécharge et dépend de la qualité de la couche et de l'interface matériau/substrat. Lorsque la couche est irradiée, la décharge est accentuée du fait que l'oxyde de plomb

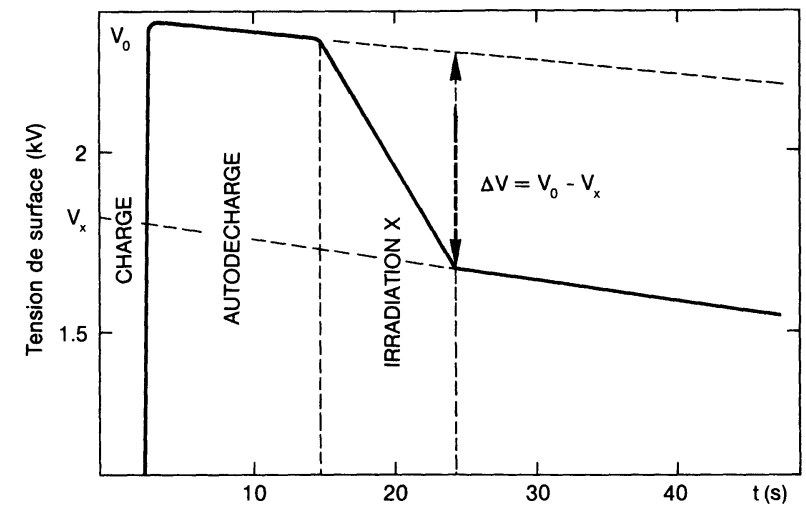

Fig. 1. - Processus de décharge xérographique : variation de la tension de surface en fonction du temps. La couche est chargée par effet Corona puis laissée se décharger dans le noir et sous irradiation. $\Delta V=V_{0}-V_{\mathrm{X}}$ : potentiel de contraste.

[Xerographic discharge : variation of the surface voltage as a function of time. Layer was charged by Corona effect and was discharged in dark and then under X-ray illumination, respectively. $\Delta V=V_{0}-V_{\mathrm{X}}$ : contrast potential.]

devient conducteur. La différence de potentiel définit un potentiel de contraste $\Delta V$ (Fig. 1).

2.2.3 Photocourant en continu. - La première électrode étant le substrat lui-même (Al ou ITO), une deuxième électrode d'or est évaporée sur la surface de la couche composite. L'échantillon est placé dans un boîtier qui l'isole électriquement de l'environnement. Le potentiel est appliqué sur une des électrodes tandis que l'autre électrode est connectée à l'entrée d'un circuit comportant une résistance et un amplificateur dont la sortie est reliée à un oscilloscope numérique à mémoire (Philips PM 3315).

\section{Résultats.}

Les deux formes $\alpha$ et $\beta$ du monoxyde ont un gap différent, respectivement à 1,9 et $2,76 \mathrm{eV}$. Les propriétés électriques et photoconductrices mesurées sur des monocristaux [6] montrent respectivement une résistivité et une mobilité de $10^{6} \Omega \mathrm{cm}$ et $100 \mathrm{~cm}^{2} / \mathrm{V} \mathrm{s}$ pour $\alpha$ et $10^{10} \Omega \mathrm{cm}$ et $50 \mathrm{~cm}^{2} / \mathrm{V} \mathrm{s}$ pour $\beta$. La résistivité atteint $10^{10} \Omega \mathrm{cm}$ pour une pastille polycristalline et $10^{16} \Omega \mathrm{cm}$ pour des couches minces préparées par évaporation $[7,2]$. La nature des porteurs dans l'oxyde de plomb reste très discutée $[6,7]$.

3.1 Traitement DeS POUdres. - Avant l'élaboration des couches composites, les poudres $\mathrm{PbO}$ subissent préalablement des traitements thermiques ainsi qu'un fort broyage par attrition. Les figures 2 et 3 présentent les diagrammes de diffraction et d'ATD caractéristiques de ces poudres.

Le traitement thermique sous $\mathrm{N}_{2}$ ne révèle pas de 


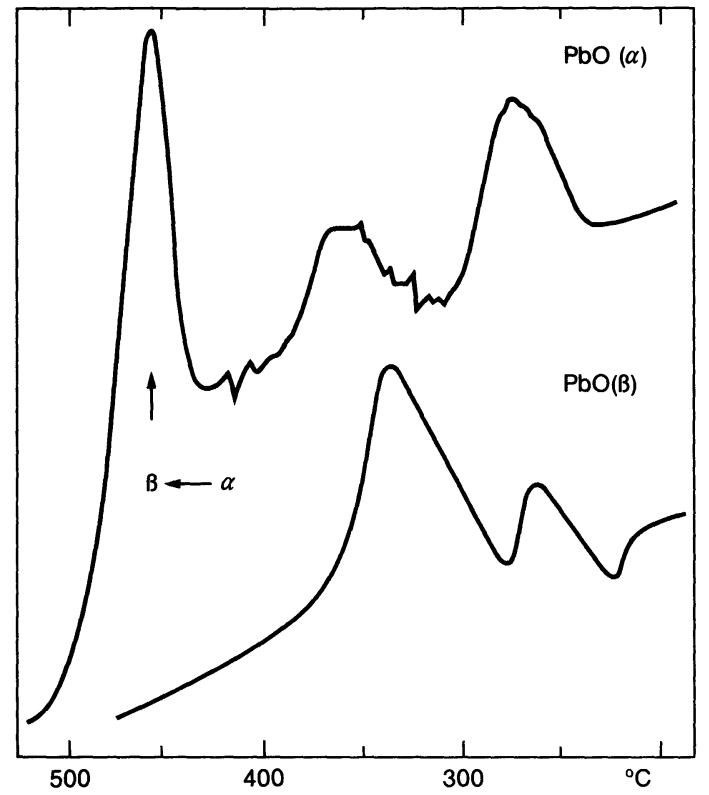

Fig. 2. - Diagramme d'analyse thermique différentielle des poudres $\mathrm{PbO} \alpha$ et des $\mathrm{PbO} \beta$.

[DTA traces of $\mathrm{PbO} \alpha$ and $\mathrm{PbO} \beta$ powders.]

changement de la structure des poudres. Par contre, les diagrammes thermiques montrent de fortes similitudes avec ceux des hydroxydes de plomb (hydrocérusite et plumbonacrite). Les pics endothermiques

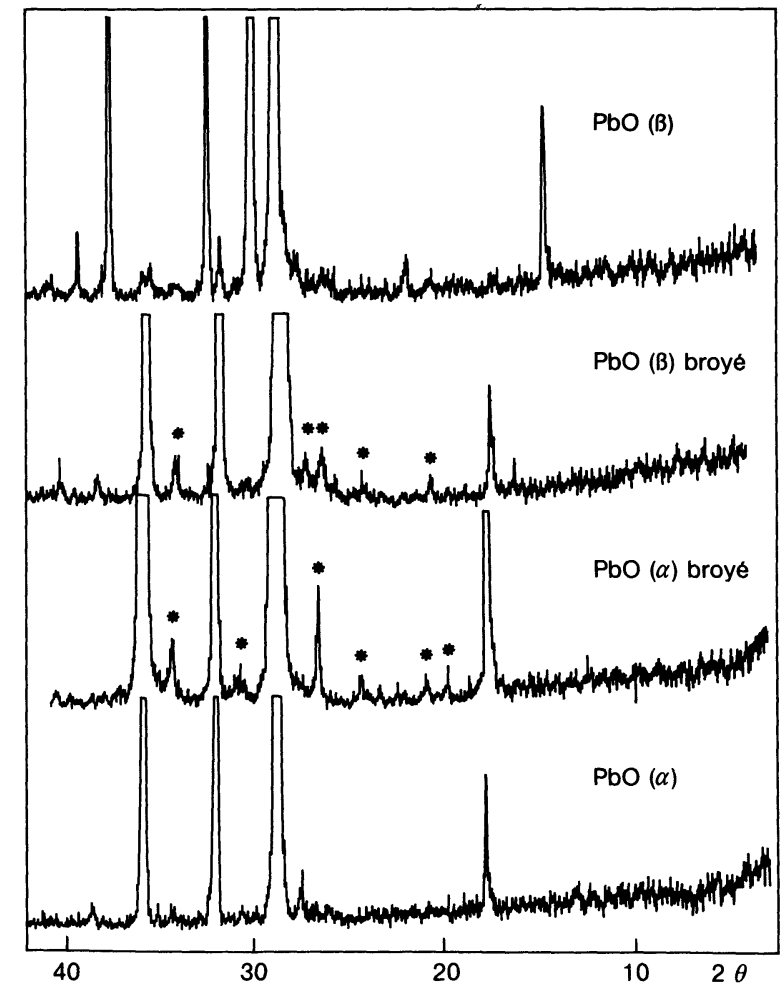

a) observés à 270,320 et à $250,300{ }^{\circ} \mathrm{C}\left(T_{\text {onsel }}\right)$ respectivement pour $\mathrm{PbO} \alpha$ et $\mathrm{PbO} \beta$ (Fig. 2) sont attribuables à la décomposition des hydroxydes de plomb présents à l'état de traces significatives à la surface des poudres. Ceci est à corréler avec l'hypothèse d'une "désorption " de l'oxygène ou plus précisément d'un départ d'espèces protonnées ainsi que cela a été déjà proposé lors de l'étude d'autres oxydes photoconducteurs comme $\mathrm{ZnO}, \mathrm{CdO}$ [9] en couches minces.

3.1.1 Sous l'action du broyage en milieu aqueux la phase $\beta$ de la poudre initiale se transforme en phase $\alpha$ (Fig. 3a). Si la poudre initiale est sous forme $\alpha$, le faible élargissement observé sur des raies de diffraction indique que le broyage n'induit qu'un léger désordre à la surface des poudres. Les poudres $\mathrm{PbO}$ obtenues par broyage se carbonatent très facilement, en accord avec la formation de défauts type hydroxyde.

3.1.2 Un dopage des oxydes de plomb par l'oxyde de titane a été réalisé dans le but de déplacer la transition $\alpha / \beta$ (habituellement à $489^{\circ} \mathrm{C}$ ) vers des températures plus hautes. Le dopage s'effectue par réaction en phase solide entre $\mathrm{PbO}$ et $\mathrm{TiO}_{2}$ à 650 $700{ }^{\circ} \mathrm{C}$. Pour un taux de $\mathrm{TiO}_{2}$ inférieur à $4 \%$ (molaire) la poudre obtenue est un mélange des deux phases $(\alpha$ et $\beta$ ) de PbO. La proportion de ces

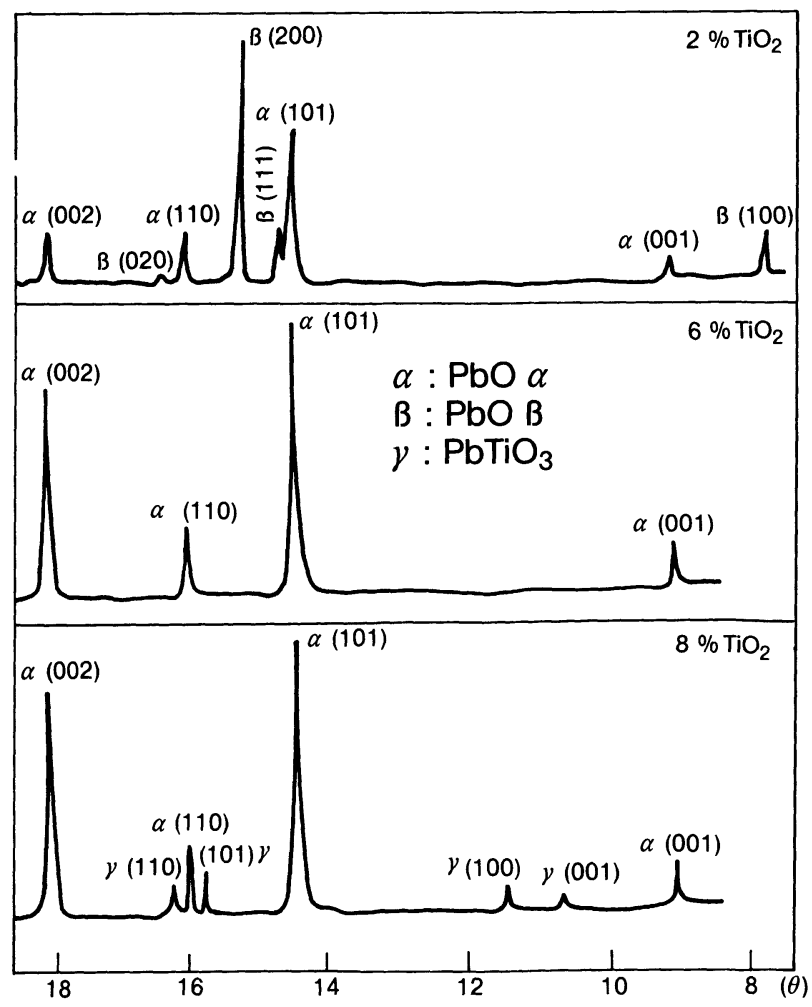

b)

Fig. 3. - Diffractogramme des poudres $\mathrm{PbO}$ ayant subi des traitements thermiques ou mécaniques (gauche) et un dopage par $\mathrm{TiO}_{2}$ (droite) (* traces de carbonate).

[Diffractogramme of $\mathrm{PbO}$ powders after thermal or mecanic treatment (left) and doping effect by $\mathrm{TiO}_{2}$ (right) (* carbonate traces).] 
deux phases est de $90 \%$ pour $\mathrm{PbO} \beta$ et $10 \%$ pour $\mathrm{PbO} \alpha$ avec un taux de $0,25 \%$ en $\mathrm{TiO}_{2}$. Pour un dopage plus important, entre 4 et $8 \%$, on obtient une solution solide entre la phase $\mathrm{PbO} \alpha$ et $\mathrm{TiO}_{2}$ et au-delà de $8 \%$ la phase $\mathrm{PbTiO}_{2}$ apparaît (Fig. 3b). On peut penser que les atomes de titane s'insèrent seulement dans la structure de la phase $\alpha$ et bloque ainsi la transition $\alpha \rightarrow \beta$. Une contraction des paramètres de maille est observée [10].

3.2 Percolation. - Les couches épaisses finales de $\mathrm{PbO}$ sont constituées de polycristaux de $\mathrm{PbO}$ sous forme de plaquettes $(5 \times 5 \times 2 \mu \mathrm{m})$ enrobées dans une résine époxy. La continuité électrique nécessite une percolation des grains de $\mathrm{PbO}$. Différentes proportions $\mathrm{PbO} /$ résine ont été réalisées pour déterminer le seuil de percolation. La figure 4 montre la variation du potentiel de contraste $(\Delta V)$ en fonction du pourcentage pondéral de l'oxyde de plomb. La valeur de $\Delta V$ qui est presque nulle à $70 \%$ (pondéral) de $\mathrm{PbO}$ augmente rapidement avec la concentration croissante de $\mathrm{PbO}$ et un palier est atteint à partir de 80-85\% d'oxyde.

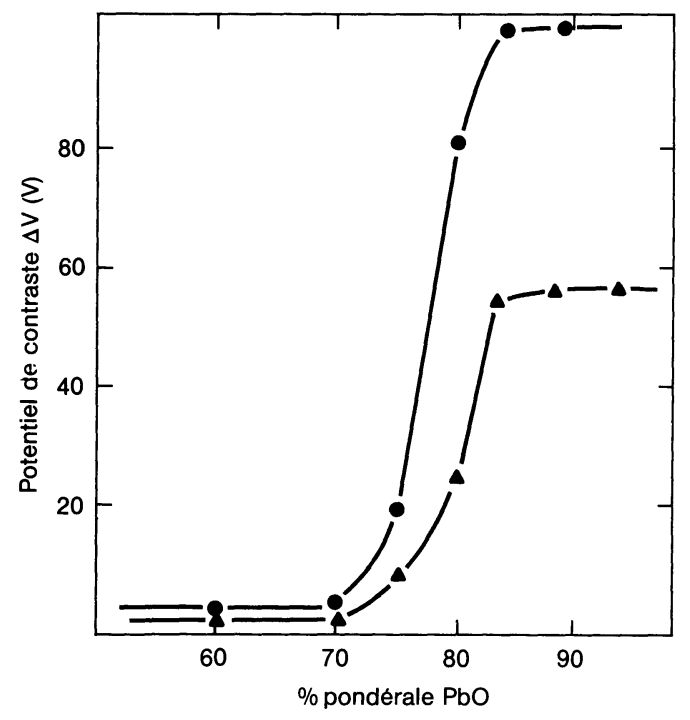

Fig. 4. - Seuil de percolation dans les couches composites $\mathrm{PbO} /$ résine époxy : variation du potentiel de contraste en fonction du pourcentage pondéral de $\mathrm{PbO}$.

[Percolation threshold in $\mathrm{PbO} /$ epoxy resin composite layer : variation of contrast potentiel as a function of $\mathrm{PbO}$ ponderal percentage.]

\subsection{CARACTÉRISTIQUES XÉROGRAPHIQUES. -} Dans les couches contenant $86 \%$ (pondéral) de $\mathrm{PbO}$, la part volumique de la résine représente $56 \%$. A une épaisseur de $200 \mu \mathrm{m}$, ces couches absorbent plus de $50 \%$ des photons $\mathrm{X}$ de $44,5 \mathrm{keV}$. Pour une même tension de pointe Corona et une même distance point-surface de l'échantillon, l'acceptance de charge $V_{0}$ augmente avec l'épaisseur des couches pour atteindre un palier à partir d'une

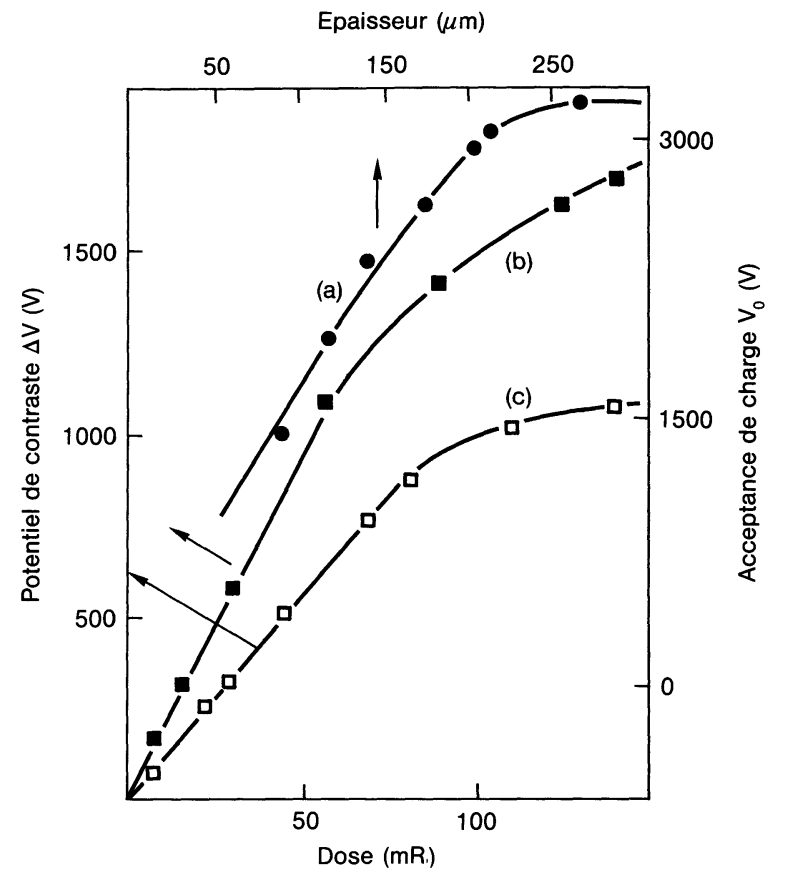

Fig. 5. - Variation de l'acceptance de charge $\left(V_{0}\right)$ (a) et du potentiel de contraste (b) en fonction de l'épaisseur de la couche composite; et du potentiel de contraste en fonction de la dose (c).

[Variation of contrast potentiel $\left(V_{0}\right)$ (a) and contrast potential (b) versus composite layer thickness; and of contrast potential versus $\mathrm{X}$ photon concentration (c).]

épaisseur de $250 \mu \mathrm{m}$ (Fig. 5). L’acceptance de charge $V_{0}$ évolue aussi en fonction du traitement préalable de la poudre de $\mathrm{PbO}$. Elle passe respectivement de 1500 à $3000 \mathrm{~V}$ lorsque les couches utilisent une poudre traitée à $310{ }^{\circ} \mathrm{C}$ sous $\mathrm{N}_{2}$.

Les couches n'ont pas le même comportement visà-vis des charges positives et négatives. L'acceptance de charge est beaucoup plus importante lorsqu'on dépose les charges négatives $(-3 \mathrm{kV})$ que des charges positives $(2 \mathrm{kV})$. La vitesse d'autodécharge est ainsi plus rapide pour les charges négatives, ce qui laisse penser que l'oxyde serait plutôt de type $p$ mais la barrière d'interface peut imposer le comportement global. Par contre, le potentiel de constraste $\Delta V$ reste le même quelle que soit la nature des charges. La variation du potentiel de contraste présentée sur la figure 5 montre que les traitements des poudres avant la mise en forme influent fortement sur les propriétés photoconductrices finales des couches. Le potentiel de contraste $\Delta V$ augmente avec la température de traitement thermique en atmosphère d'azote. La température optimum se situe vers $310{ }^{\circ} \mathrm{C}$ (Tab. I).

L'évolution du potentiel de contraste en fonction de la concentration des photons $\mathrm{X}$ incidents est linéaire à faible dose puis atteint un palier à partir d'une valeur seuil (Fig. 5). L'écoulement des charges de la surface vers l'électrode arrière n'est pas 
complet. Tout se passe comme si un champ électrique s'établissait et s'opposait à cette évacuation. Il s'agit probablement d'une polarisation induite par le courant des photoporteurs. Par ailleurs, des mesures successives sur la même couche montrent une décroissance de $\Delta V$ en fonction du nombre de cycles de mesure. Si on suppose que la chute du potentiel de surface lors de l'irradiation $\mathrm{X}$ ne dépend que de la couche photoconductrice (en négligeant les effets d'interfaces) on peut estimer la quantité des charges déplacées par la relation : $Q=\Delta V \times C$ ( $C$ étant la capacité de la couche et $\Delta V$ le potentiel de contraste). De cette relation on peut déduire un rendement $S$ représentant le nombre d'électrons créés par photon incident. Le tableau I résume les rendements obtenus pour les couches composites. Le meilleur résultat concerne une couche de $200 \mu \mathrm{m}$ contenant $83 \%$ de $\mathrm{PbO}$ traités à $310{ }^{\circ} \mathrm{C}$ sous azote.

Les modifications structurales de l'oxyde, induites par le broyage, affectent les propriétés photoconductrices des couches. Le potentiel de contraste $\Delta V$ de la couche contenant $\mathrm{PbO} \alpha$ obtenu par broyage de $\mathrm{PbO} \beta$ est de $100 \mathrm{~V}$. Celui de la couche contenant $\mathrm{PbO} \alpha$ broyé est de $50 \mathrm{~V}$ au lieu des $85 \mathrm{~V}$ obtenus avant le traitement de la poudre. La photosensibilité de la phase $\alpha$ est plus importante que celle de la

Tableau I. - Acceptance de charge, potentiel de contraste et rendement des couches composites (83\% en $\mathrm{PbO} ; e=250 \mu \mathrm{m})$.

[Charge acceptance, contrast potential and efficiency of composite layers $(83 \% \mathrm{PbO}, e=250 \mu \mathrm{m})$.

\begin{tabular}{|cc|c|r|r|}
\hline \multicolumn{2}{|c|}{$N_{\mathrm{p}}=2 \times 10^{8} / \mathrm{cm}^{2}$} & \multicolumn{1}{c|}{$V_{0}$} & $\Delta V$ & $S$ \\
\hline PbO $(\alpha)$ & & 1100 & 85 & 173 \\
traité & $200 \% \mathrm{~N}_{2}$ & 1020 & 100 & 180 \\
& $310 \% \mathrm{~N}_{2}$ & $2700\left(^{*}\right)$ & 324 & 605 \\
& & 1400 & 200 & 375 \\
& $410 \% \mathrm{~N}_{2}$ & 1200 & 154 & 268 \\
& $460 \% \mathrm{~N}_{2}$ & 1100 & 71 & 133 \\
$\mathrm{PbO}(\alpha)$ broyé & 1356 & 50 & 93 \\
$\mathrm{PbO}(\beta)$ broyé & 1450 & 100 & 175 \\
$\mathrm{Se}(1000$ ppm As) & 2000 & 279 & \\
$\mathrm{Se}(100$ ppm As) & 1450 & 197 & \\
\hline
\end{tabular}

(*) Couche de $300 \mu \mathrm{m}$ avec une concentration de $86 \%$ en $\mathrm{PbO}$.

$N_{\mathrm{p}}$ : nombre de photons incidents par $\mathrm{cm}^{2}, V_{0}$ : acceptance de charge ; $\Delta V$ : potentiel de contraste, $S$ : rendement exprimé par le nombre d'électrons collectés par photon $\mathrm{X}$ incident.

(*) Layer of $300 \mu \mathrm{m}$ thickness containing $86 \% \mathrm{PbO}$.

$N_{\mathrm{p}}$ : number of incident photons per $\mathrm{cm}^{2} . V_{0}$ : charge acceptance ; $\Delta V$ : contrast potential ; $S$ : number of collected electrons per one absorbed $\mathrm{X}$ incident photon. phase $\beta$, ce qui explique l'augmentation de $\Delta V$ de la couche contenant du $\mathrm{PbO} \beta$ broyé. La diminution de $\Delta V$ des poudres $\mathrm{PbO} \alpha$ broyées est due à l'apparition des espèces protonnées $(\mathrm{O}-\mathrm{H})$ et/ou de carbonates à la surface des poudres $\mathrm{PbO}$ broyées. Ceci est en accord avec le gain de photosensibilité obtenu par le traitement thermique des poudres éliminant ainsi les défauts et conduisant sans doute à une structure de surface des poudres plus favorable à la photoconductivité.

3.4 PROPRIÉtÉs ÉleCTRIQUES. - Les mesures de courant d'obscurité et de photo-courant ont été réalisées sous l'irradiation de photons visibles ou de photons X de $44,5 \mathrm{keV}$. L'évolution temporelle ainsi que les dépendances photo-courant/champ électrique et photo-courant/puissance lumineuse sont similaires quelle que soit la nature des photons incidents.

3.4.1 Evolution temporelle du photo-courant.- La figure 6 présente la réponse temporelle du photocourant pour différents champs électriques (positifs) appliqués. Dans le cas de $\mathrm{PbO}$, jusqu'à une valeur de champ seuil $E_{\mathrm{s}}$, le photo-courant présente un pic, puis une décroissance suivie d'un palier $\left(i_{\mathrm{p}}\right)$. Pour des champs supérieurs à $E_{\mathrm{s}}$ le courant croît après le pic pour ne se stabiliser qu'après un temps assez long (quelques heures). La valeur de ce palier est très supérieure à $i_{\mathrm{p}}$ ( 2 ordres de grandeur).

Cette évolution est comparable à celle observée pour $\mathrm{Bi}_{12} \mathrm{GeO}_{20}$, autre matériau photoconducteur $[11,12]$. La phase décroissante du courant est due probablement à l'établissement d'un champ de polarisation dans le sens contraire du champ appliqué. Ce champ interne est induit par une répartition des charges d'espace dans le volume des matériaux. Lorsque le champ appliqué est important le champ interne est négligeable et de ce fait on n'observe plus

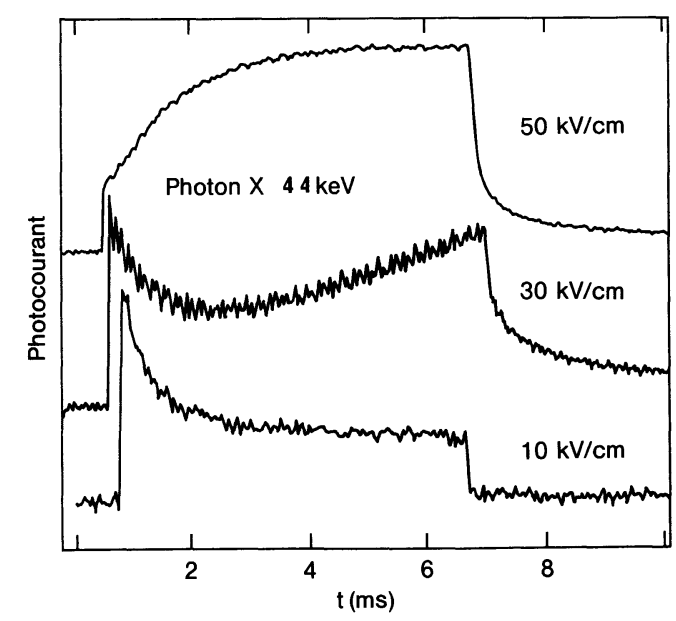

Fig. 6. - Evolution temporelle du photo-courant à différents champs électriques appliqués.

[Temporal evolution of photo-current at different electric field.] 
le pic. La phase croissante peut être expliquée par un phénomène d'injection des électrons de l'électrode vers le matériau. En effet, si le contact est bloquant l'injection n'est possible que si l'amplitude des perturbations au niveau de l'électrode est supérieure à la barrière de potentiel qui sépare l'electrode et l'échantillon. C'est le cas où le champ appliqué dépasse le champ seuil. L'évolution de ce champ seuil en fonction de la puissance lumineuse est décroissante. Il en est de même pour la dépendance puissance seuil/champ électrique. La quantité de charges éjectées par unité de temps provoquant l'injection des électrons est donc indépendante de la puissance lumineuse et du champ appliqué.

$\mathrm{Si}$ on irradie de nouveau la couche $\mathrm{PbO}$ après une première irradiation (le champ électrique étant absent) il apparaît alors un courant qui circule dans le sens inverse du photo-courant comme si un champ négatif existait entre les électrodes. Ce phénomène indique que l'échantillon a été polarisé lors de la première excitation par le déplacement des charges d'espace à l'intérieur de la couche photoconductrice. Les charges dépolarisées c'est-à-dire celles collectées lors de la deuxième irradiation en absence de champ, dépendent du champ appliqué lors de la mesure de photo-courant et de la sensibilité du matériau. Elles atteignent une valeur de saturation $Q_{\mathrm{s}}$ de l'ordre de $5 \times 10^{-9} \mathrm{C} / \mathrm{cm}^{2}$ pour une couche de $150 \mu \mathrm{m}$ d'épaisseur ( $\mathrm{PbO}$ traitée à $\left.310{ }^{\circ} \mathrm{C}\right)$.

3.4.2 Evolution du courant d'obscurité et du photocourant. - La variation du courant de fuite en fonction du champ présente deux régimes. A faible champ, le courant varie linéairement : le contact est ohmique et la résistivité est de l'ordre de $10^{12} \Omega \mathrm{cm}$. A fort champ, le courant varie de façon exponentielle comme si le contact était injectant. Le comportement du photo courant est analogue à celui du courant de fuite. Les charges collectées lors de l'irradiation varient exponentiellement avec le champ (Fig. 7). Une dépendance non linéaire du photo-courant en fonction du champ a déjà été observée pour les couches minces de $\mathrm{PbO}$ préparées par évaporation [2]. Ceci est en accord avec l'hypothèse d'un courant de charges d'espace dans les couches compliquant ainsi les mesures du photocourant.

Les propriétés électriques des couches sont aussi affectées par les traitements préalables des poudres. L'augmentation de la température du recuit thermique sous azote entraîne une augmentation du photocourant et une diminution du courant de fuite (Fig. 8). L'optimum de la photosensibilité est obtenu avec un traitement à $310{ }^{\circ} \mathrm{C}$. Dépassant cette température le courant de fuite augmente de nouveau et le photo-courant rediminue. Ces résultats sont similaires à ceux des poudres $\mathrm{PbO}$ compactées [13] ou à ceux d'autres matériaux contenant du plomb [9].

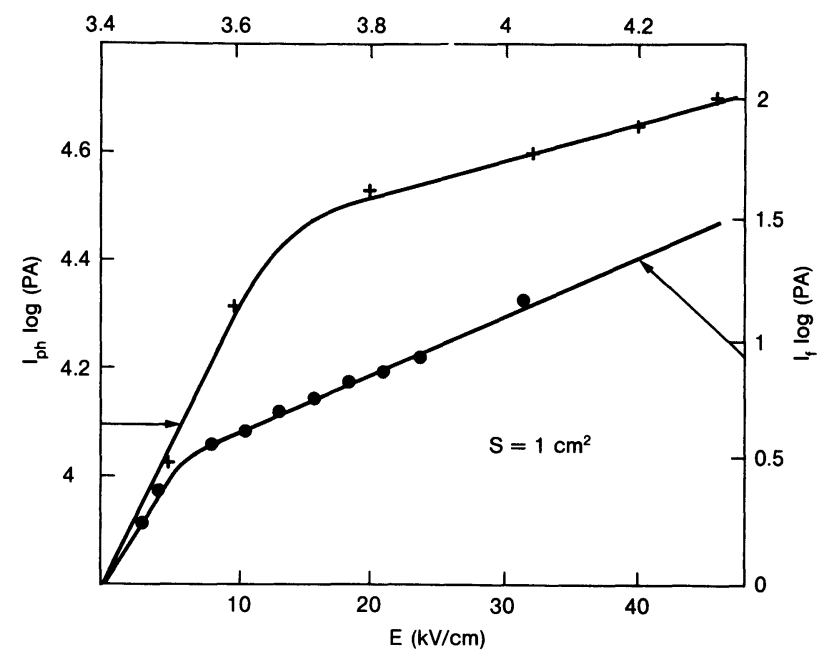

Fig. 7. - Variation du courant d'obscurité $(\bullet)$ et du photo-courant $(+)$ en fonction du champ électrique pour des couches composite contenant $\mathrm{PbO} \alpha$.

[Variation of dark-current $(\bullet)$ and photo-current $(+)$ versus electric field for composite layer containing $\mathrm{PbO} \alpha$.

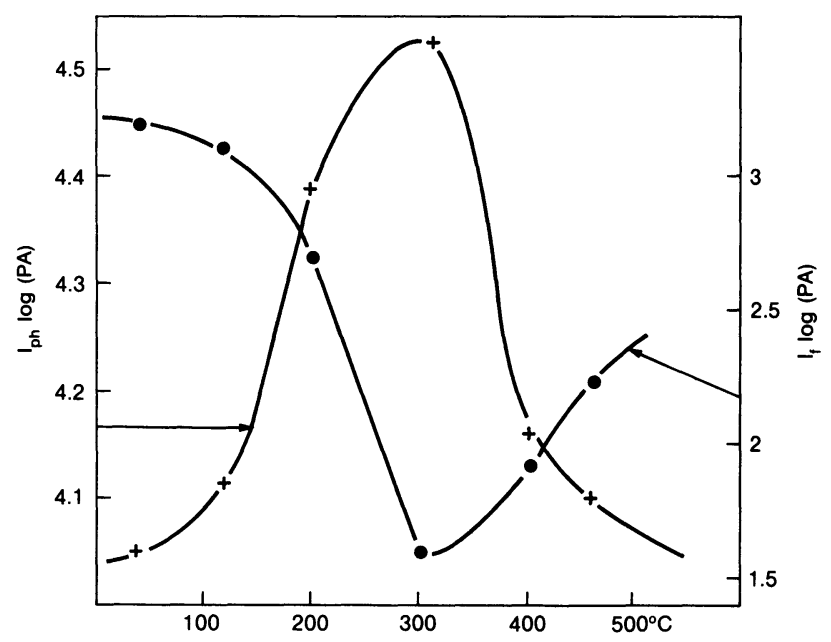

Fig. 8. - Evolution du courant d'obscurité (•) et du photo-courant en fonction de la température du traitement préalable de la poudre de $\mathrm{PbO} \beta$.

[Dark current $(\bullet)$ and photo-current $(+)$ evolution versus $\mathrm{PbO} \boldsymbol{\beta}$ powder treatment temperature.]

Comme nous l'avons vu avec la décharge xérographique, le broyage affaiblit la photosensibilité de la poudre $\mathrm{PbO}(\alpha)$ tandis que le traitement thermique sous atmosphère neutre l'améliore nettement. Cet effet est toujours à relier à l'absorption d'espèces protonnées $\left(\mathrm{OH}^{-}, \mathrm{H}_{2} \mathrm{O} \ldots\right)$ lors du broyage puis à leur départ lors du traitement thermique.

En ce qui concerne les poudres $\mathrm{PbO}$ dopées avec $\mathrm{TiO}_{2}$, le nombre d'électrons collectés par photons $\mathrm{X}$ incident varie avec le taux de dopage. Cette sensibilité est maximale pour un taux de $0,25 \%$ en $\mathrm{TiO}_{2}$ puis décroît pour des taux supérieurs (Fig. 9) 

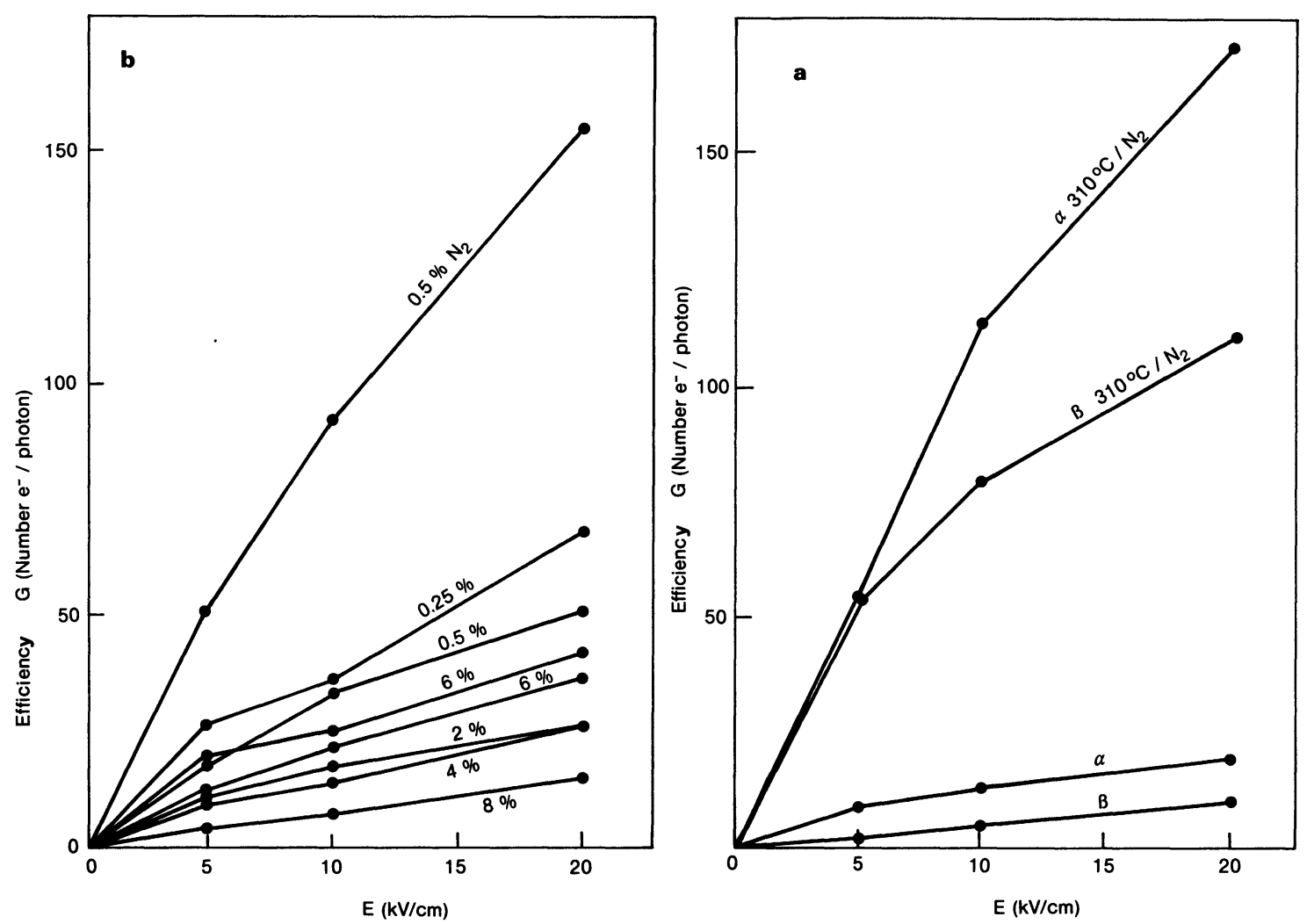

Fig. 9. - Efficacité des couches composites (nombre d'électrons collectés par photon $\mathrm{X}$ incident) : (a) couche contenant la poudre $\mathrm{PbO}$ traitée thermiquement; (b) couche contenant la poudre $\mathrm{PbO}$ dopée au $\mathrm{TiO}_{2}$.

[Composite layer efficiency : (number of $\mathrm{e}^{-}$collected per photon incident ; (a) layer containing PbO powder, (b) layer containing $\mathrm{PbO}$ powder doped with $\mathrm{TiO}_{2}$.]

avec l'apparition de modifications structurales. A $8 \%$ de $\mathrm{TiO}_{2}$ la sensibilité de la poudre dopée devient même inférieure à celle de $\mathrm{PbO} \alpha$ pur. Le traitement thermique sous atmosphère neutre augmente la sensibilité, toutefois pour une poudre dopée de $0,5 \%$ cette sensibilité reste intermédiaire entre celles des phases $\mathrm{PbO} \alpha$ et $\mathrm{PbO} \beta$ pures traitées dans les mêmes conditions. La structure restant la même, on peut penser que le dopage affecte principalement les porteurs électriques.

La photosensibilité des couches en fonction du champ appliqué a été évaluée en reportant le rapport $i_{\text {photo-courant }} / i_{\text {obscurité. }}$ Par exemple pour $\mathrm{PbO} \alpha$ dopé $0,5 \%$ en $\mathrm{TiO}_{2}$ la sensibilité augmente avec le champ et atteint un maximum pour $E=30 \mathrm{kV} / \mathrm{cm}$ puis décroît. On constate que ce gain maximum correspond à la limite d'existence d'une loi ohmique entre champ électrique appliqué et courant d'obscurité. Il est donc nécessaire de connaître la valeur de ce champ pour définir les conditions de fonctionnement des dispositifs.

\section{Conclusion.}

Cette étude montre que les couches composites $\mathrm{PbO} /$ résine déposées par sérigraphie possèdent une bonne qualité de surface, une bonne homogénéité d'épaisseur et peuvent conduire à la réalisation d'écrans photoconducteurs $X$. La percolation se situe à un pourcentage pondéral voisin de $82 \%$ en oxyde de plomb sur des poudres de dimensions voisines de $5 \times 5 \times 2 \mu \mathrm{m}$. Ces couches ont une bonne tenue sous fort champ électrique, une importante résistivité dans l'obscurité $\left(10^{12}-10^{13} \Omega \mathrm{cm}\right)$ et un rendement acceptable de 605 électrons collectés par photon $\mathrm{X}$ incident $\left(44,5 \mathrm{keV}, \quad N=2 \times 10^{8}\right.$ photon $\left./ \mathrm{cm}^{2}, \quad V_{0}=2,7 \mathrm{kV} / \mathrm{cm}\right)$. Ce rendement est largement supérieur à celui d'une couche usuelle à base de sélénium dopée à l'arsenic.

La caractérisation électrique et photoconductrice a mis en évidence l'importance des phénomènes d'interface et de polarisation. Le contact a un comportement ohmique à faible voltage et injectant à fort voltage. Le changement de comportement électrique se situe pour des champs électriques supérieurs à $10 \mathrm{kV} / \mathrm{cm}$. Les charges de dépolarisation ne sont pas négligeables et atteignent un seuil de saturation vers $6 \times 10^{-9} \mathrm{C}$ (soit $20 \%$ des photosporteurs créés)

L'activation préalable de la poudre (traitements thermiques, broyage) joue un rôle crucial sur la photosensibilité des couches. Les évolutions du 
courant de fuite, du photo-courant lors du chauffage sous atmosphère neutre ou lors du broyage en milieu aqueux sont cohérentes et comparables à celles observées pour d'autres photoconducteurs comme
$\mathrm{PbS}, \mathrm{ZnO}$ ou les composés ternaires contenant du plomb [9]. L'évolution de ces courants est due à la désorption des espèces oxygénées $\left(\mathrm{O}_{2}, \mathrm{OH}^{-}\right)$pendant le traitement thermique.

\section{Bibliographie}

[1] Heijne L., Philips Res. Suppl. 4 (1961);

Powell M. H. and PetTs R. C., Opt. Lett. 11 (1986) 36.

[2] Clarke J. R., Greene J. E., Thin Solid Films 66 (1980) 339 ;

VAN Der DrIfT A., Philips Res. Rept. 22 (1967) 267.

[3] Kwestroo W., Huizing A., J. Inorg. Nucl. Chem. 27 (1965) 1951.

[4] Clasen R., J. Photographic Sci. 28 (1980) 226.

[5] Boher P., Garnier P., J. Solid State Chem. 55 (1984) 245.

[6] Keezer R. C., Bowman D. L., Becker J. H., $J$. Appl. Phys. 39 (1968) 2062.

[7] Izvozchikov V. A., Avanesyan V. T., Bor-
DovskII B. A., BordovskiI G. A., Ferroelectrics 18 (1987) 105.

[8] Izvozchiкov V. A., Phys. Status. Solidi (a) 14 (1972) 161.

[9] Bube R. H., Photoconductivity of Solids (John Wiley \& Sons) 1967.

[10] Moreau J., Boher P., Garnier P., Mat. Res. Bull. 24 (1989) 1241.

[11] RichaRd B., Thèse Paris (1987).

[12] Oberschmid R., Phys. Status Solidi (a) 89 (1985) 657.

[13] Radhakrishnan S., Kamalasanau N. J., MehenDRU P. C., J. Mater. Sci. 18 (1983) 1912. 